Statistik 2011 der Kinderschutzgruppe und Opferberatungsstelle des Kinderspitals Zürich

\title{
Wieder hohe Zahl von gemeldeten Fällen von Kindsmisshandlung
}

\section{Ulrich Lips}

Leiter Kinderschutzgruppe und Opferberatungsstelle
Korrespondenz: Dr. med. Ulrich Lips Leiter Kinderschutzgruppe und Opferberatungsstelle Universitätskinderkliniken Steinwiesstrasse 75 CH-8032 Zürich Tel. 0442667318

ulrich.lips[at]kispi.uzh.ch www.kinderschutzgruppe.ch
Die Anzahl der gemeldeten Fälle von gesicherter oder vermuteter Kindsmisshandlung stagniert auf hohem Niveau: Im Jahr 2011 hat die Kinderschutzgruppe und Opferberatungsstelle des Kinderspitals Zürich 484 Meldungen erhalten, nur drei weniger als im Rekordjahr 2010. Die Zunahme der Fälle, die letztes Jahr als Zufallseffekt interpretiert werden konnte, scheint sich nun als Trend zu bestätigen. Deutlich zugenommen im Vergleich zum Vorjahr
Deutlich wird, dass die Gesamtzahl der Meldungen wegen gesicherter oder vermuteter sexueller Ausbeutung von 33\% im Jahre 2010 auf 40,2\% im Jahr 2011 angestiegen ist (von 162 auf 195 Fälle). Dabei sind deutlich mehr Knaben betroffen (2010: 29 / 2011: 65).

Auch von körperlicher Misshandlung sind mehr Knaben betroffen (2010: 70 / 2011: 94), während die Gesamtzahl ungefähr gleich geblieben ist. Es muss

\section{«Die Fehleinschätzung, eine Anzeige bei der Polizei löse automatisch eine erneute Traumatisierung des Opfers aus, verliert immer mehr an Boden.»}

hat der Anteil Meldungen wegen sexueller Ausbeutung, wobei mit 70\% (2010: 37\%) deutlich mehr Opfer den Schritt unternahmen, einen gesicherten Übergriff der Polizei zu melden. Der Anteil minderjähriger Verursacher von sexuellen Übergriffen hat von $32 \%$ auf $26 \%$ abgenommen.

\section{Die Zahlen im Detail}

Abbildung 1 zeigt die im Jahr 2011 gemeldeten Misshandlungsformen im Vergleich zum Vorjahr. aber offen bleiben, ob wirklich mehr Knaben Opfer von Misshandlungen geworden sind oder ob mehr Knaben, respektive ihre Eltern es wagen, sich als Opfer zu «outen», eine Entwicklung, die bei erwachsenen Männern schon seit einer Weile zu beobachten ist. Die prozentualen Verschiebungen bei den anderen Misshandlungsformen sind unwesentlich und wohl teilweise darauf zurückzuführen, dass sich diese Formen stark überschneiden und nicht immer gleich klassifiziert werden. Zu erwähnen ist leider

Abbildung 1

Misshandlungsmeldungen der Jahre 2010 und 2011.

\begin{tabular}{|c|c|c|c|}
\hline & & 2011 \\
\hline
\end{tabular}


Abbildung 2

Meldungen von (Verdacht auf) Kindsmisshandlung im Kinderspital Zürich 1992 bis 2011.

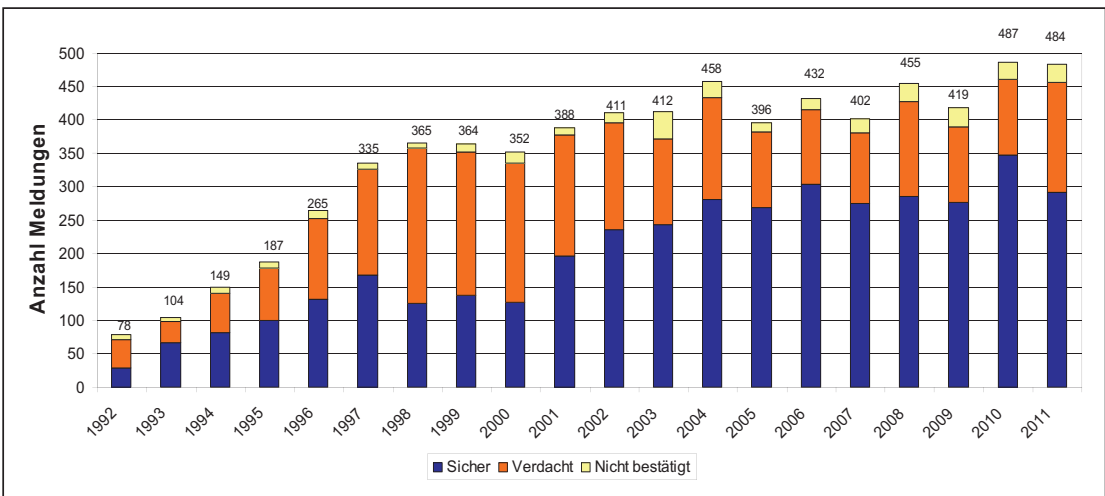

auch, dass zwei Kinder an der Folge der Misshandlung gestorben sind. Eine Gesamtübersicht über die Meldungen der letzten 20 Jahre zeigt Abbildung 2.

\section{Weniger minderjährige Täter - deutlich mehr Opfer melden einen Übergriff der Polizei}

Unsere Zahlen zeigen eindeutig, dass die Zahl der minderjährigen Täter sowohl relativ als auch in Absolutzahlen abnimmt. Dies verdeutlicht Abbildung 3.

Markant verändert hat sich auch das Verhalten der Opfer nach einem sexuellen Übergriff: Schon immer hatte die Kinderschutzgruppe in einer solchen Situation zu einer Strafanzeige geraten, im Jahre 2010 folgten jedoch nur 37\% der Opfer eines gesicherten sexuellen Übergriffs diesem Rat. 2011 hingegen machten 70\% der Opfer eine Meldung an die Polizei. Das zeigt, dass sich die gute Arbeit und die einfühlsame Befragung der besonders dafür ausgebildeten Polizeibeamtinnen und -beamten herumspricht. Die Fehleinschätzung, eine Anzeige bei der Polizei löse automatisch eine erneute Traumatisierung des Opfers aus, verliert immer mehr an Boden.

\section{Abbildung 3}

Alter und Geschlecht der Täterinnen und Täter in gesicherten Fällen von sexueller Ausbeutung 2007 bis 2011.

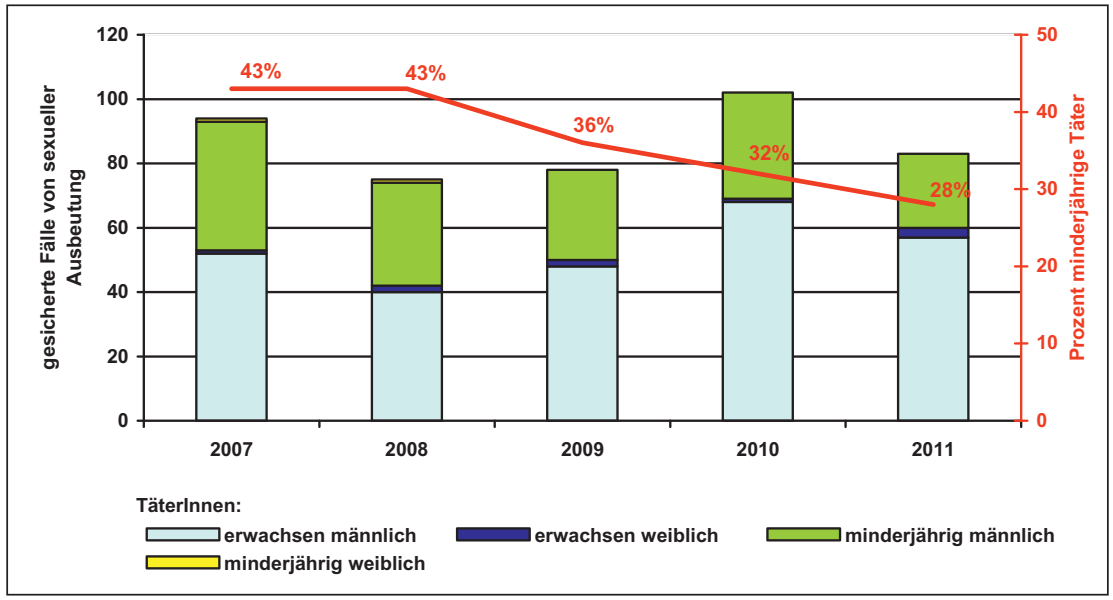

Über die Kinderschutzgruppe und Opferberatungsstelle des Kinderspitals Zürich

Die Kinderschutzgruppe befasst sich mit Säuglingen, Kindern und Jugendlichen, die gesichert oder mutmasslich Opfer einer Misshandlung wurden oder gefährdet sind, misshandelt zu werden. Ziel der Kinderschutzgruppe ist es, durch sorgfältig geplante Interventionen drohende Misshandlungen abzuwenden und betroffene Kinder und Jugendliche vor wiederholter Misshandlung zu schützen. Im Zentrum der Aktivitäten steht das Wohl der Kinder und Jugendlichen, für die möglichst optimale Bedingungen für die weitere Entwicklung geschaffen werden sollen. Die interdisziplinäre Arbeitsweise mit Spezialistinnen und Spezialisten aus Medizin, Psychiatrie, Psychologie, Gynäkologie und Sozialarbeit ermöglicht, die verschiedenen Facetten einer Misshandlungs-Situation zu erfassen und entsprechend zu reagieren. Bezugspersonen sowie nachbehandelnde und nachkontrollierende Institutionen werden früh in die Arbeit und Entscheide der Kinderschutzgruppe einbezogen.

Meldungen gesicherter oder vermuteter Misshandlungen gelangen über die folgenden Personengruppen oder Institutionen an die Kinderschutzgruppe:

- betroffene Kinder und Jugendliche,

- deren Familien, Angehörige oder Bekannte,

- Fachleute, Institutionen und Behörden, die mit Kindern, Jugendlichen und deren Familien arbeiten.

Nach den Vorgaben des Opferhilfegesetzes erhalten Opfer einer strafbaren Handlung Beratung und Unterstützung in rechtlichen, psychosozialen und unter bestimmten Voraussetzungen auch in finanziellen Belangen.

\section{Beratungen von Fach- und Bezugspersonen weiterhin gefragt}

Auch 2011 wandten sich viele Fach- und Bezugspersonen (Grosseltern, Nachbarn, Lehrpersonen, Hausund Kinderärztinnen und -ärzte, Behördenmitglieder usw.) für eine Beratung wegen einer Misshandlungssituation an uns (insgesamt 243 / 2010: 240). Dies kann auch als Hinweis darauf gewertet werden, dass die Öffentlichkeit vermehrt Fachhilfe sucht, wenn Anzeichen für eine Kindsmisshandlung vorhanden sind, und nicht die Augen verschliesst.

\section{Leitfaden zur Früherfassung von Kindsmiss- handlung durch Ärztinnen und Ärzte}

Die Broschüre «Kindsmisshandlung - Kinderschutz. Ein Leitfaden zu Früherfassung und Vorgehen in der ärztlichen Praxis» (www.kinderschutz. ch) erschien im April 2011 und wurde an 20000 Ärztinnen und Ärzte verschiedener Fachgebiete in der Schweiz verschickt. 